\title{
DETECCIÓN SÉRICA DE PSA MEDIANTE UN TEST RÁPIDO (SD BIOLINE PSA)
}

Francisco Serrano de la Cruz Torrijos, Emilio López Alcina, Joaquín Ulises Juan Escudero, Milagros Fabuel Deltoro, Ana Montoliu García, Arcadio Real Romaguera', Macarena Ramos de Campos y Emilio Marqués Vidal.

Servicio de Urología. Consorcio Hospital General Universitario de Valencia. Valencia. España. 'Profesor Asociado de la Escuela de Enfermería. Universidad de Valencia. Valencia. España.

Resumen.- OBJETIVO: En los últimos años han aparecido numerosos test semicuantitativos para la determinación de PSA, basados en la inmunocromatografía, realizados sobre suero o plasma. Presentamos nuestra experiencia en el uso del test SD BIOLINE PSA, que se realiza en plasma o suero para determinación cualitativa de PSA de forma rápida, y que usa como punto de corte $3 \mathrm{ng} / \mathrm{ml}$.

MÉTODO: Se analizaron un total de 54 pacientes que estuvieron ingresados en nuestra sala de hospitalización. Se extrajeron dos muestras de sangre a todos los paciente una para determinación cuantitativa de PSA en el laboratorio de nuestro hospital y otra para la determinación cualitativa con el test SD BIOLINE PSA, poste- riormente comparamos ambos resultados. Dos urólogos interpretaron de forma independiente el test sin conocer los valores de PSA obtenidos en laboratorio. Para calcular el efecto del tiempo de lectura del test se leyó a los 15, 20 y 25 minutos. Los resultados fueron clasificados en una tabla de contingencia clásica, lo cual nos permitió calcular la sensibilidad y especificidad del test, asi como el valor predictivo positivo y el valor predictivo negativo.

RESULTADOS: La edad media fue de 71.1 años (rango de 43-96 años). De los 54 pacientes sometidos al estudio 26 (48. 14\%) tuvieron un PSA $>3 \mathrm{ng} / \mathrm{mL}$ (media $18,5 \mathrm{ng} / \mathrm{mL}$, rango $3.9-66.9 \mathrm{ng} / \mathrm{mL}$ ) y 28 (51.86\%) presentaron un PSA $<3 \mathrm{ng} / \mathrm{mL}$ (media 0.8 $\mathrm{ng} / \mathrm{mL}$, rango $0-2.9 \mathrm{ng} / \mathrm{mL}$ ), en el análisis ordinario. Los resultados en cada intervalo y por observador fueron los siguientes: 15 minutos: Observador 1: sensibilidad (S) 76.92\%, especificidad (E) 100\%, valor predictivo positivo (VPP) 100\%, valor predictivo negativo (VPN) 82.35\%; Observador 2: S 76.92\%, E 100\%, VPP 100\%, VPN 82.35. 20 minutos: Observador 1: S 100\%, E 93.33\%, VPP 92.30\%, VPN 100\%; Observador 2: S 100\%, E 93.33\%, VPP 92.30\%, VPN 100\%. 25 minutos: Observador 1: S 100\%, E 85.71\%, VPP 86.66\%, VPN 100\%; Observador 2: S 92.30\%, E $92.85 \%$, VPP $92.30 \%$, VPN $92.85 \%$.

CONCLUSIONES: El test SDBioline cumple las características necesarias para ser utilizado como prueba de detección del PSA, es simple, rápido, barato, poco invasivo y presenta una buena efectividad.

Palabras clave: PSA. Cribaje. Diagnóstico rápido. Cáncer de próstata. 
Summary.- OBJECTIVES: Over the last years numerous semiquantitative PSA tests have appeared, based on serum or plasma immunochromatography. We present our experience using the SD BIOLINE PSA test, which is performed with plasma or serum for fast qualitative determination of PSA; the cutpoint is $3 \mathrm{ng} / \mathrm{ml}$.

METHODS: We analized 54 patients who were admitted in our hospital ward. Two blood samples were obtained from every patient, one for cuantitative PSA determination at the hospital laboratory and the other one for qualitative determination with the SD BIOLINE PSA test, and the results were compared.. Two urologists independently interpreted the test without knowing the PSA values from the lab. To calculate the effect of test reading time, readings were performed at 15,20 and 25 minutes. Results were classified in a classic contingency table, which enabled us to calculate sensitivity and specificity of the test, as well as positive and negative predictive values.

RESULTS: Mean age was 71.1 years (range 43-96 yr). From 54 patients in the study 26 (48.14\%) had a PSA $>3 \mathrm{ng} / \mathrm{ml}$ (Mean $18.5 \mathrm{ng} / \mathrm{ml}$, range $3.9-66.9 \mathrm{ng} / \mathrm{ml}$ ) and $28(51.86 \%$ ) PSA $<3 \mathrm{ng} / \mathrm{mL}$ (mean $0.8 \mathrm{ng} / \mathrm{mL}$, range $0-2.9 \mathrm{ng} / \mathrm{mL}$, in the conventional test. Results for each interval and observer were: $15 \mathrm{~min}$ : Observer 1: Sensitivity (S) 76,92\%, specificity (E) 100\%, positive predictive value (PPV) $100 \%$, negative predictive value (NPV) 82.35\%; Observer 2: S 76.92\%, E 100\%, PPV 100\%, NPV 82.35\%. 20 minutes: Observer 1: S 100\%, E 93.33\%, PPV 92.30\%, NPV 100\%; Observer 2: S 100\%, E 93.33\%, PPV 92.30\%, NPV 100\%. 25 minutes: Observer 1: S 100\%, E 85.71\%, PPV 86.66\%, NPV 100\%; Observer 2: S 92.30\%, E 92.85\%, PPV $92.30 \%$, NPV $92.85 \%$.

CONCLUSIONS: The SD Bioline PSA test complies with the characteristics required to be used as a test for prostate cancer detection, it is simple, fast, cheap, not much invasive, and has a good efficacy.

Keywords: PSA. Screening. Fast diagnosis. Prostate cancer.

\section{INTRODUCCIÓN}

El cáncer de próstata es el segundo tumor en frecuencia, por detrás del superficial de piel en varones más de 50 años. Al año se diagnostican alrededor de 85.000 nuevos casos en toda Europa, lo que supone un $13 \%$ de todas las neoplasias. Su incidencia aumenta un $3 \%$ anualmente.

A partir de los 50 años la incidencia aumenta de forma rápida, pudiendo llegar a afectar a más de la mitad de la población masculina. En estudios necrópsicos a varones, se diagnosticó latente un carcinoma de próstata en más del $30 \%$ de los varones mayores de 50 años, aumentando al $40-50 \%$ en los mayores de 70 años.

La probabilidad de que se desarrolle un carcinoma de próstata en hombres menores de 40 años es de 1 entre 10.000, entre los 40-59 años de 1 entre 1.000 y entre los 60-79 años es de 1 entre 8.

La tendencia al aumento en su incidencia se debe al envejecimiento de la población, a la mejora en el diagnóstico (se detectan tumores que podrían haber pasado desapercibidos) y al creciente interés por el cribaje por parte de la población.

La alta prevalencia y la posibilidad de diagnóstico precoz apoyarían el cribaje, así como el aumento significativo de diagnóstico de tumor localizado y la reducción de la enfermedad metastásica (1).

Estudios recientes parecen indicar un descenso de la mortalidad, gracias a una combinación de factores, entre ellos el diagnóstico precoz basado en el cribaje de PSA (1).

Las principales controversias (1) antes de establecer un cribaje masivo son:

- Sobrediagnóstico: ya que se diagnostican tumores que nunca producirían morbimortalidad.

- Coste económico del tratamiento y el de las complicaciones.

La determinación del PSA es usada ampliamente para identificar hombres con elevado riesgo de cáncer de próstata (3). Se necesita un test de screening de PSA similar al del embarazo o de la microalbuminuria, debería ser un test fácil de usar, que no necesite equipamiento especial y que aporte un resultado de forma rápida (4).

En los últimos años han aparecido numerosos test semicuantitativos de determinación de PSA, basados en inmunocromatografía, realizados sobre suero o plasma.

Presentamos nuestra experiencia en el uso del test SD BIOLINE PSA, que se realiza en plasma o suero para determinación cualitativa de PSA de 
forma rápida y que utiliza como punto de corte 3 $\mathrm{ng} / \mathrm{ml}$.

\section{MATERIAL Y MÉTODOS}

\section{Pacientes y muestras}

Incluimos en nuestro estudio un total de 60 pacientes, 54 hombres y 6 mujeres, que estuvieron ingresados en nuestra sala de hospitalización independientemente de la patología que justificaba el ingreso y de los antecedentes personales. Para determinar el número mínimo de pacientes en los que realizar la determinación de PSA se utilizó la técnica de la media progresiva observando que 50 determinaciones eran suficientes para no obtener cambios significativos en la media. Los pacientes fueron seleccionados al azar.

Se obtuvieron dos muestras de sangre a todos los paciente, tras otorgar su consentimiento, una para determinación cuantitativa de PSA en el laboratorio de nuestro centro y otra para la determinación cualitativa con el test SD BIOLINE PSA, posteriormente procedimos a la comparación de ambos resultados.

Se tomaron como grupo control 6 mujeres, a quienes se les sometió a un estudio similar.

\section{Fundamentos del test}

El test SD BIOLINE PSA consiste en una inmunocromatografía in vitro que con un paso permite determinar de forma cualitativa el PSA en suero o plasma. El punto de corte del test es de $3 \mathrm{ng} / \mathrm{ml}$.

El test contiene una membrana recubierta de anticuerpos anti-PSA de ratón en la banda del test. La muestra de suero se mueve a través de la membrana y en la región $T$ del test forma una línea visible, por el complejo anticuerpo-antígeno-anticuerpo.

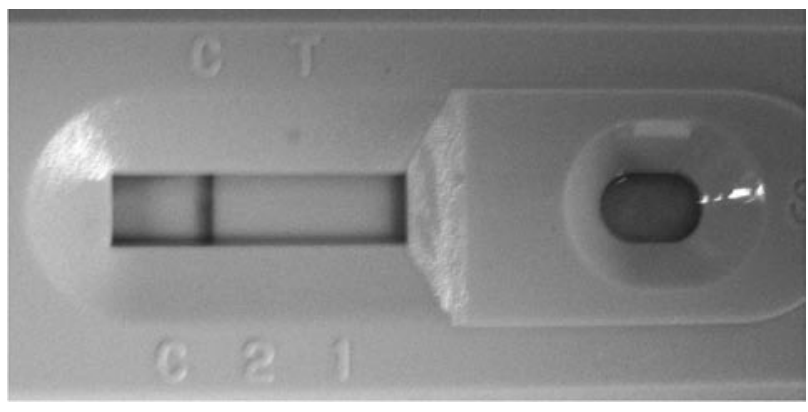

FIGURA 1. Negativo: si sólo aparecía una banda de color en la ventana del test.

\section{Proceso e interpretación del test}

Añadimos una gota de suero o plasma en la cazoleta del test. Para obtener la muestra de plasma procedíamos a la centrifugación de la muestra de sangre, y la de suero la conseguíamos tras dejar coagular la muestra una hora. Por comodidad hemos usado muestra de suero, ya que no precisa de ningún instrumento adicional, tan sólo dejar la sangre en un tubo de muestra sin anticoagulantes.

Posteriormente realizamos la lectura del test a los $15-20$ y 25 minutos. El resultado podía ser:

1) Negativo: si sólo aparecía una banda de color en la ventana del test (Figura 1).

2) Positivo: si aparecían dos bandas de color en la ventana del test (Figura 2).

3) Nulo: cuando no se observaba ninguna banda. Algunas causas de esto pueden ser una mala realización del test o un deterioro del mismo (Figura 3).

Dos urólogos interpretaron de forma independiente el test sin conocer los valores de PSA obtenidos en laboratorio. Para calcular el efecto del tiempo de lectura del test se leyó a los 15, 20 y 25 minutos. Los resultados fueron analizados en una tabla de contingencia clásica, tomando como patrón el resultado obtenido en el laboratorio, lo cual nos permitió calcular la sensibilidad y especificidad del test, así como el valor predictivo positivo y el valor predictivo negativo.

\section{RESULTADOS}

La edad media fue de 71.1 años (rango de 43-96 años). De los 54 pacientes sometidos al estudio $26(48.14 \%$ ) tuvieron un PSA $>3 \mathrm{ng} / \mathrm{mL}$ (media $18,5 \mathrm{ng} / \mathrm{mL}$, rango $3.9-66.9 \mathrm{ng} / \mathrm{mL}$ ) y $28(51.86 \%)$

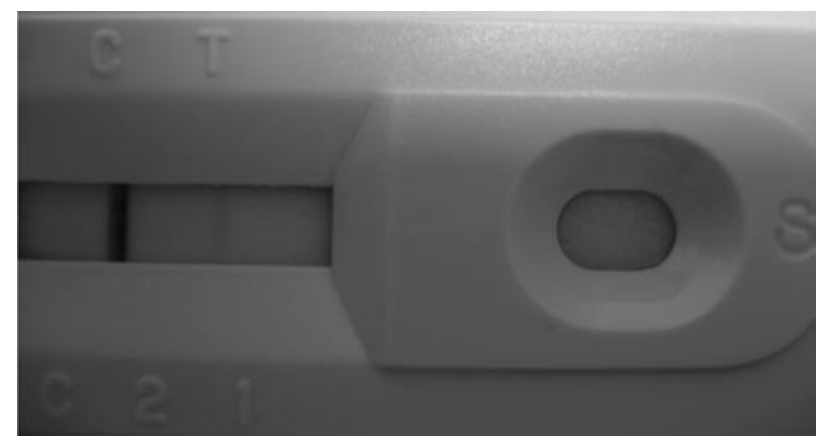

FIGURA 2. Positivo: si aparecían dos bandas de color en la ventana del test. 


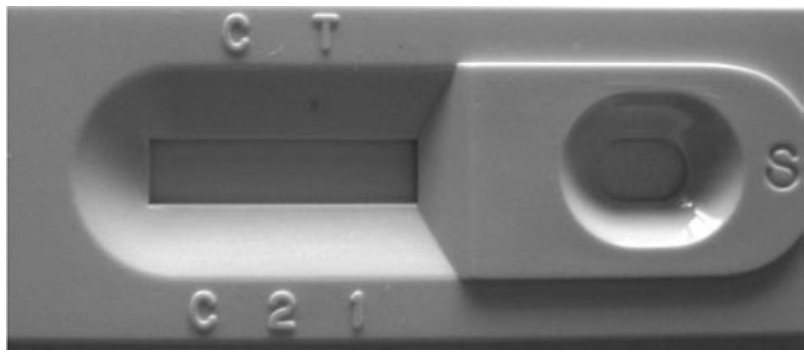

FIGURA 3. Nulo: cuando no se observaba ninguna banda. Algunas causas de esto pueden ser una mala realización del test o un deterioro del mismo.

presentaron un PSA $<3 \mathrm{ng} / \mathrm{mL}$ (media $0.8 \mathrm{ng} / \mathrm{mL}$, rango $0-2.9 \mathrm{ng} / \mathrm{mL}$ ), en el análisis de laboratorio.

Los resultados obtenidos en el test al igual que la variabilidad interobservador se vieron afecta- dos por el tiempo transcurrido hasta su lectura, presentando los mejores resultados y concordancia entre observadores a los 20 minutos de inicio del test.

Los resultados en cada intervalo y por observador se detallan en la Tabla I.

En el grupo control de 6 mujeres todas presentaron PSA de $0 \mathrm{ng} / \mathrm{mL}$, obteniendo con el test en los tres casos resultado negativo, a los 15,20 y 25 minutos.

\section{DISCUSIÓN}

Según datos provenientes del registro de mortalidad de la Comunidad Valenciana el cáncer de próstata supone una media anual de 445 muertes, situándolo como el segundo tumor masculino. Esto implica una tasa de mortalidad del 22 por 100.000 habitantes en nuestro medio.

\section{TABLA I.}

\begin{tabular}{|c|c|c|}
\hline \multicolumn{3}{|l|}{ A) 15 minutos: } \\
\hline & OBSERVADOR 1 & OBSERVADOR 2 \\
\hline SENSIBILIDAD & $76.92 \%$ & $76.92 \%$ \\
\hline ESPECIFICIDAD & $100 \%$ & $100 \%$ \\
\hline VPP & $100 \%$ & $100 \%$ \\
\hline VPN & $82.352 \%$ & $82.35 \%$ \\
\hline \multicolumn{3}{|l|}{ B) 20 minutos: } \\
\hline & OBSERVADOR 1 & OBSERVADOR 2 \\
\hline SENSIBILIDAD & $100 \%$ & $100 \%$ \\
\hline ESPECIFICIDAD & $93.33 \%$ & $93.33 \%$ \\
\hline VPP & $92.30 \%$ & $92.30 \%$ \\
\hline VPN & $100 \%$ & $100 \%$ \\
\hline \multicolumn{3}{|l|}{ C) 25 minutos: } \\
\hline & OBSERVADOR 1 & OBSERVADOR 2 \\
\hline SENSIBILIDAD & $100 \%$ & $92.30 \%$ \\
\hline ESPECIFICIDAD & $85.71 \%$ & $92.85 \%$ \\
\hline VPP & $86.66 \%$ & $92.30 \%$ \\
\hline VPN & $100 \%$ & $92.85 \%$ \\
\hline
\end{tabular}

VPP: Valor Predictivo Positivo. VPN: Valor Predictivo Negativo. 
Las herramientas más usadas en el diagnóstico del cáncer de próstata son el tacto rectal, el PSA y la ecografía transrectal (5). El diagnóstico de certeza siempre es anatomopatológico y nos aporta también el grado del tumor. Si una de estas pruebas es positiva el riesgo de biopsia positiva es del $6-25 \%$, si dos pruebas son positivas el riesgo aumenta al $18-60 \%$; y si son las tres positivas el riesgo asciende al $56-72 \%(5)$.

EI PSA es una calicreína producida por las células epiteliales de la próstata que tiene la función de licuar el semen. Se puede elevar en numerosas situaciones, como HBP, prostatitis y cáncer, es órgano específico pero no cáncer específico (4). El aumento de su nivel sérico no siempre indica cáncer de próstata, aunque sí alteración prostática y precisa de un estudio del paciente.

Aunque el cribaje para cáncer de próstata es uno de los más controvertidos, está reconocido que el tacto rectal y el PSA, usados juntos, son las mejores herramientas para su diagnóstico (1), siendo el PSA el mejor predictor (4-5), ya que ha contribuido al aumento del diagnóstico de tumores órgano-confinados y a la disminución de la mortalidad.

Los programas de cribaje de gran calado como el proyecto "pívot" del Nacional Cáncer Institute y el programa Europeo de Cáncer que intentan reclutar 74000 y 50000 hombres respectivamente están aún en vías de desarrollo y tendremos que esperar a 2010 para disponer de resultados sólidos y con ello la posibilidad de validar el cribaje en cáncer de próstata.

La medida del PSA ha sido establecida como un método efectivo para ayudar a detectar cáncer de próstata. El cribaje se debería usar para un inicio precoz del tratamiento, aunque su valor no es todavía decisivo (2). No obstante, hemos de contemplar situaciones particulares en varones mayores de 40 años asintomáticos que tengan un riesgo elevado de padecer la enfermedad. En concreto en aquellos pacientes con familiares de primer grado afectos de cáncer de próstata con un elevado riesgo añadido parece plenamente indicado el cribaje.

Otra situación a considerar es la facilidad de acceso de los varones a pruebas de detección rápida de PSA (medios de información de masas como Internet, clínicas o farmacias) por lo que parece oportuno evaluar la efectividad de estos medios de diagnóstico en condiciones de uso habitual en los centros hospitalarios.

Si se solventan las controversias del cribaje de cáncer de próstata (alto coste, posibilidad de so- bretratamiento, consenso en la mejor modalidad de terapéutica) los programas para ello estarían ampliamente aceptados. (1)

Un programa de screening sería aceptable por los pacientes si fuera simple, no invasivo y rápido; la aceptación por parte del Servicio Nacional de Salud depende de dos características, su bajo coste y su efectividad (1). El precio de una determinación aislada de PSA ronda los $20 €$, siendo el coste total del test SD Bioline aproximadamente la mitad. Tras la utilización del test que nos ocupa, podemos asegurar que es simple, ya que sólo necesita agregar unas gotas de plasma o suero al dispositivo, siendo su lectura sencilla, como ya se ha explicado anteriormente. En cuanto a la invasividad, tan sólo se necesita la extracción de sangre por venopunción, lo cual no es una agresión importante para el paciente. En lo referente a la rapidez del resultado es donde se puede presentar algún inconveniente, ya que al utilizar suero - plasma necesitamos dejar coagular o centrifugar la muestra de sangre respectivamente; una vez iniciado el test, el resultado lo tendremos en 20 minutos, momento en el cual hemos obtenido los mejores resultados. Dado que la utilidad del test es para el screening, es más importante la sensibilidad que la especificidad (4). El test SD Bioline PSA ha mostrado una sensibilidad del $100 \%$ y una especificidad del 93.33\% respecto a la determinación clásica, siendo el punto de corte $3 \mathrm{ng} / \mathrm{mL}$, por lo cual aceptamos la buena efectividad del dicho test.

Comentar en relación con los niveles de PSA en nuestro estudio que no son representativos, ni era lo pretendido, de la población general a la que atendemos, dado que se trata de pacientes ingresados en sala, muchos de ellos de edad avanzada y portadores de sonda, motivo por el cual un porcentaje elevado $(48 \%)$ presentaba un valor superior a $3 \mathrm{ng} / \mathrm{mL}$.

El test evaluado detecta un punto de corte de PSA de $3 \mathrm{ng} / \mathrm{mL}$ lo que parece más indicado que otros tests con puntos de corte superiores dada la tendencia actual a adoptar una postura determinante en los programas de detección precoz de la enfermedad.

Los resultados son equiparables o incluso mejores que los que ofrecen otros test similares (Tabla II).

Un resultado positivo en el test implica la necesidad de realizar una medición cuantitativa de PSA (1-4). De cualquier modo, con un resultado negativo en el test, siempre debería asociarse un tacto rectal para excluir una potencial sospecha de cáncer de próstata (1). 
TABLA II.

\begin{tabular}{|c|c|c|c|c|}
\hline AUTOR & TEST PSA & MUESTRA & SENSIBILIDAD & ESPECIFICIDAD \\
\hline Jung y cols. & Chembio & Suero & $67 \%$ & $87 \%$ \\
\hline Jung y cols. & Medpro & Suero & $87 \%$ & $88 \%$ \\
\hline Jung y cols. & Syntron & Suero & $93 \%$ & $93 \%$ \\
\hline Jung y cols. & Seratec & Suero & $80 \%$ & $97 \%$ \\
\hline Dok An y cols. & One step PSA & Suero & $100 \%$ & $90 \%$ \\
\hline Madersbacher y cols. & Oncoscreen & Suero & $93 \%$ & $93 \%$ \\
\hline Berg y cols. & Uralen & Sangre capilar & $91 \%$ & $81 \%$ \\
\hline Miano y cols. & PSA RapidScreen & Sangre capilar & $97.6 \%$ & $90.4 \%$ \\
\hline
\end{tabular}

\section{CONCLUSIONES}

El test SDBioline PSA cumple las características necesarias para ser utilizado como screening del cáncer de próstata, es simple, rápido, barato, poco invasivo y presenta una buena efectividad. De cualquier forma, precisa ser validado de forma externa, con estudios multicéntricos, y encontraría su principal lugar en el cribaje del cáncer de próstata si éste se demostrase eficiente.

\section{NOTA DE LOS AUTORES}

Manifestar que no tenemos vinculación profesional o económica con el distribuidor pero agradecemos que nos haya suministrado gratuitamente los kits de detección para el presente estudio.

\section{BIBLIOGRAFIA y LECTURAS RECOMENDADAS ( ${ }^{*}$ lectura de interés $y^{* *}$ lectura fundamental)}

**1. MIANO, R.; MELE, G.: "Evaluation of a new, rapid, qualitative, one-step Test for prostate cancer screening: The PSA Rapid Screen test". Prostate Cancer and Prostatic Diseases, 8: 219, 2005.
*2. CHANG DOK, A.N.; YOSHIKI, T.: "Evaluation of a rapid qualitative prostate specific antigen assay, the One Step PSA test". Cancer Lett., 162: $135,2001$.

*3. OBERPENNING, F.; HETZEL, S.: "Semi-quantitative immunochromatographic Test for Prostate Specific Antigen in Whole Blood: Tossing the Coin to Predict Prostate Cancer?". European Urology, 43: 478, 2003.

*4. JUNG, K.; ZACHOW, J.: "Rapid detection of elevated prostate-specific antigen levels in blood: Performance of various membrane strip tests compared". Urology, 53: 155, 1999.

*5. GUIDELINES DE CANCER DE PRÓSTATA. 2007.

6. LOERTZER, H.; FISCHER, K.; FORNARA, P.: "PSA quick test in capillary blood". Anticancer Res., 25: 1659, 2005.

7. MADERSBACHER, S.; MIAN, C.; MAIER, U. y cols.: "Validation of a 10 minute dipstick test for serum prostate specific antigen". Eur. Urol., 30: 446, 1996.

8. BERG, W.; LINDER, C.; ESCHHOLZ, G. y cols.: "Pilot study of the practical relevance of a onestep test for PSA in capillary blood to improve the acceptance rate in the early detection program of prostate carcinoma". Int. Urol. Nephrol., 32: 381, 2001. 